\title{
Qualitatively Different Hippocampal Subfield Engagement Emerges with Mastery of a Spatial Memory Task by Rats
}

\author{
Guillaume L. Poirier, Eman Amin, and John P. Aggleton \\ School of Psychology, Cardiff University, Cardiff CF10 3AT, United Kingdom
}

The parallel, entorhinal cortex projections to different hippocampal regions potentially support separate mnemonic functions. To examine this possibility, rats were trained in a radial-arm maze task so that hippocampal activity could be compared after "early" (two sessions) or "late" (five sessions) learning. Induction of the immediate-early gene Zif268 was then measured, so revealing possible activity differences across hippocampal subfields and the parahippocampal cortices. Each rat in the two experimental groups (early, late) was also yoked to a control rat that obtained the same number of rewards, visited the same number of maze arms, and spent a comparable amount of time in the maze. Although overall Zif268 levels did not distinguish the four groups, significant correlations were found between spatial memory performance and levels of dentate gyrus Zif268 expression in the early but not the late training group. Conversely, hippocampal fields CA3 and CA1 Zif268 expression correlated with performance in the late but not the early training group. This reversal in the correlation pattern was echoed by structural equation modeling, which revealed dynamic changes in effective network connectivity. With early training, the dentate gyrus appeared to help determine $\mathrm{CA1}$ activity, but by late training the dentate gyrus reduced its neural influence. Furthermore, CA1 was distinguished from CA3, each subfield developing opposite relations with task mastery. Thus, functional entorhinal cortex coupling with CA1 activity became more direct with additional training, so producing a trisynaptic circuit bypass. The present study reveals qualitatively different patterns of hippocampal subfield engagement dependent on task demands and mastery.

Key words: effective connectivity; network; immediate-early gene; Zif268; hippocampal subfields; radial-arm maze

\section{Introduction}

Models of hippocampal organization have traditionally been based on the serial pathway from the entorhinal cortex to the dentate gyrus (DG), hippocampal field CA3, and, thence, to hippocampal field CA1. The realization that layer III entorhinal cortex neurons project directly to CA1 and the subiculum, adjacent to the layer II entorhinal neurons that project densely on the dentate gyrus (Steward and Scoville, 1976; Witter et al., 2000), has transformed our understanding of hippocampal organization. These parallel, entorhinal projections suggest that the dentate gyrus and CA1 could have independent memory functions. Proposals include the idea that the dentate gyrus is more important for the encoding of spatial information, whereas CA1 is more critical for consolidation or retrieval processes (Lee and Kesner, 2004; Remondes and Schuman, 2004). Much of the evidence for this view has come from lesion studies (for review, see Kesner et al., 2004), along with some support from computational modeling and physiological evidence (McClelland and Goddard, 1996; Redish and Touretzky, 1997; Lörincz and Buzsáki, 2000; Rolls and Kesner, 2006). There are, however, inherent limitations in testing this proposal with the lesion approach. It is not only tech-

Received July 30, 2007; revised Dec. 10, 2007; accepted Dec. 14, 2007

This work was funded by the Medical Research Council (UK). We thank Dr. Gordon T. Harold, Dr. Kate L. Shires, and Cédric Ginestet for their assistance and the anonymous reviewers for their comments.

Correspondence should be addressed to Guillaume L. Poirier, School of Psychology, Cardiff University, 70 Park Place, Cardiff CF10 3AT, UK. E-mail: PoirierG@cardiff.ac.uk.

D01:10.1523/JNEUROSCI.4607-07.2008

Copyright $\odot 2008$ Society for Neuroscience $\quad$ 0270-6474/08/281034-12\$15.00/0 nically difficult to make selective lesions and spare all fibers of passage, but any dentate gyrus/CA3 lesion will also partially disconnect CA1 and so may alter the normal responsivity of CA1 cells.

The solution is to compare functional contributions across an intact brain. For this reason, the present study used immediateearly gene (IEG) imaging. The IEG zif268 (also known as egr-1 or krox24) was studied because its expression is associated with both spatial memory formation and long-term plasticity in the hippocampus, rather than neural activity per se (Wisden et al., 1990; Richardson et al., 1992; Abraham et al., 1993, 1994; Herdegen and Leah, 1998; Hughes et al., 1999; Tischmeyer and Grimm, 1999; Guzowski, 2002; Davis et al., 2003; Lindecke et al., 2006; Kubik et al., 2007).

The present study examined whether the dentate gyrus, CA3, and CA1 show differential patterns of Zif268 activity across spatial learning. The study used the radial-arm maze variant that taxes both working and reference memory by having a subset of never-baited arms (Olton and Papas, 1979). Because the task constrains behavioral parameters such as arm choice and the number of arm entries, yoked animals provide close behavioral controls. Furthermore, this radial-maze task not only provides clear measures of spatial learning within and across sessions, but is sensitive to dorsal hippocampal lesions (Pothuizen et al., 2004). To remove the contribution from learning the task rules, rats were pretrained in a radial-arm maze in a separate room (Bannerman et al., 1995; Cain, 1998). The study also used structural equation modeling to evaluate network dynamics by deriving 


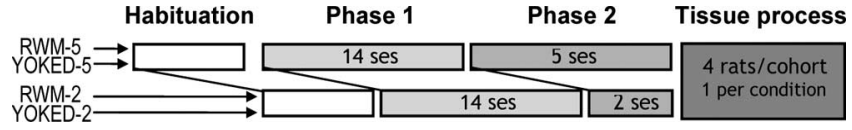

Figure 1. Schematic plan of training protocols and timetable. Rats were habituated to a maze in room one (habituation) and then trained for 14 sessions (14 ses) on a combined working and reference memory task in a radial-arm maze (phase 1). Next, the rats were trained in a new maze in a second room (phase 2). Group RWM-5 received five sessions (5 ses), whereas group RWM-2 received two sessions (2 ses). Each experimental rat was "yoked" to a control rat (YOKED-5 or YOKED-2). Thus, each cohort consisted of one rat from each of the four conditions, and each cohort finished testing on the same day by staggering the start of habituation.

estimates of the influence of one site over another (McIntosh and Gonzalez-Lima, 1991; Friston et al., 1993; Jenkins et al., 2003). Consequently, the current study is potentially the first to use IEG activity to test the causal relations between any brain regions according to the level of mastery of a spatial task.

\section{Materials and Methods}

Subjects

The subjects were 32 Dark Agouti rats (Harlan, Bicester, Oxfordshire, $\mathrm{UK}$ ) kept in a $13 \mathrm{~h}$ light/11 h dark cycle with ad libitum access to water. The rats were food restricted and maintained at $85 \%$ of their free-feeding weight throughout the experiment. They were housed in pairs, one experimental rat with its yoked control. All rats were habituated to handling before start of the experiment, and the cohort divided into four groups of eight rats. All experiments were performed in accordance with the UK Animals (Scientific Procedures) Act (1986) and associated guidelines.

\section{Apparatus}

The apparatus consisted of an elevated radial maze (Olton and Samuelson, 1976). Eight arms ( $87 \mathrm{~cm}$ long $\times 10 \mathrm{~cm}$ wide) were symmetrically arranged around an octagonal central platform $(34 \mathrm{~cm}$ diameter). The floor of the maze was made of laminated wood, and the wall of the arms were made of clear Perspex $24 \mathrm{~cm}$ high. At the end of each arm was a $2 \mathrm{~cm}$ diameter food well, $0.5 \mathrm{~cm}$ deep. The walls of the central platform were formed by eight Perspex doors connected to a pulley system, thus enabling the experimenter to control the rat's access to the arms.

Testing took place sequentially in two rooms, each with a radial-arm maze of the same dimensions. Both rooms were distinctive in shape and size, and contained different salient cues (such as furniture and posters on the walls).

\section{Behavioral training}

Four groups of rats (each $n=8$ ) were compared, and throughout training rats were tested in cohorts of four, with one rat from each group (Fig. 1). Two groups learned the radial-maze task ("early" = RWM-2, "late" = RWM-5), and two groups served as their respective controls (YOKED-2, YOKED-5). All animals were initially habituated to the first test environment and shaped to seek sucrose pellets ( $45 \mathrm{mg}$; Noyes Purified Rodent Diet; Noyes, Lancaster, NH) at the end of the arms. Pairs of animals (an experimental animal with its yoked control) together underwent two 15 min habituation sessions in the apparatus, followed the next day by an additional two sessions in which the animals individually explored the apparatus until all sucrose pellets had been retrieved.

All training was in two phases, pretraining (phase 1) and test (phase 2). For phases 1 and 2, rats in the RWM groups were trained on a combined working and reference memory task (Olton and Papas, 1979). The combined reference and working memory task (Olton and Papas, 1979) was chosen because it taxes memory both within a session (working) and across sessions (reference). At the start of a trial, four of the eight arms were baited. A rat was placed in the central platform and all arms opened simultaneously to allow the rat to explore the arms and retrieve the sucrose pellets. An arm was considered selected when both hindpaws of the rat passed the threshold of the door into the arm. The doors were then all closed, and the door of the currently explored arm only reopened when the rat reached the end of the arm. After return to the central platform, after eating the food reward, all the doors were kept closed for 10 s. Access was next given to all eight arms again, each trial continuing until all four baited arms had been visited or $10 \mathrm{~min}$ had elapsed. The same four arms were baited throughout training for a given RWM rat, different sets of four arms being chosen for separate rats.

Daily training sessions consisted of four consecutive trials, and the RWM rats and their yoked counterparts all received a total of 14 complete sessions during phase 1 . For phase 2 ("test"), the rats were trained in a new room for either two (RWM-2) or five (RWM-5) daily sessions. The start of training in phase 1 was staggered by three sessions to ensure that all four rats in a cohort (one each from RWM-2, YOKED-2, RWM-5, and YOKED-5) reached their final session and were perfused on the same day (Fig. 1).

The yoked control rats were only allowed to explore four different predetermined arms per session, one arm at a time. The number of arm entries was equal to that of the rat to which it was yoked. Consequently, if a RWM rat entered more than four different arms, its yoked control would enter only four different arms, but have the appropriate number of repeat visits (an occurrence that was most frequent during early training). Furthermore, memory errors produced by the experimental animal were mirrored by presenting the yoked control with an unbaited arm for each error made. The set of four arms visited by the yoked control rats changed each session (to prevent learning the baited set), but on the final session the unbaited four arms were the same as those used for the RWM rats. In this way, yoked rats were matched as closely as possible for the total number of arm entries, the number of rewarded and nonrewarded arm entries, the duration of the exploration in the maze, and also, especially in the final test session, for the identity of the arms explored and, thus, the cues experienced. To reduce the use of intramaze cues, the maze was rotated by the angle of one arm after each trial. For the trained subjects, the positions of the baited and unbaited arms remained constant with respect to room (extramaze) cues.

\section{Behavioral parameters}

Two measures were used to characterize the learning and performance of the RWM-2 and RWM-5 rats: arm entry errors and time to retrieve all rewards. The former parameter comprised two categories: (1) reference memory errors, as defined by an entry into a nonrewarded arm; (2) working memory errors, as defined by reentry into an arm previously visited during the same trial. Two types of working memory errors were distinguished: (2a) reentry into a previously rewarded arm ["working (nonreference)" errors] and (2b) reentry into a never-rewarded arm ["working (reference)" errors]. This distinction in working memory errors was made because their causes should differ, as should their incidence levels, especially with additional training (cf. Pothuizen et al., 2004).

Several behavioral measures of learning were used to correlate with Zif268 reactivity. These measures comprised the numbers of the errors in the final test session (immediately before tissue sampling), the total number of errors in the second maze, and also the learning rate, produced by fitting a line to error scores across sessions. These rate scores enabled us to test the hypothesis that Zif268 expression is not only related to immediate performance but may also reflect an integration of previous performance. For example, this approach could help distinguish and interpret Zif268 levels of two rats presenting equivalent performance scores on the final session, but for which improvement relative to the previous session is minimal for one but substantial for the other.

\section{Immunohistochemistry}

Dragunow and Faull (1989) have cautioned that the history of the organism in the preceding 1-12 h may influence c-Fos levels at the time of sampling, thereby highlighting the critical importance of appropriate controls. Immediately after a rat's radial-arm maze session (typically lasting $\sim 10 \mathrm{~min}$ for the final session), it was placed in a dark, quiet room, made familiar over previous habituation sessions. This period ended 90 min after the onset of the radial-arm maze session. The timing of tissue sampling $(90 \mathrm{~min}$ ) was carefully based on evidence that Zi268 protein induction reaches its maximal levels $\sim 1-2 \mathrm{~h}$ after induction by various types of stimulation such as visual, tactile, and direct electrical stimulation, including that associated with hippocampal long-term potentiation 
(Gass et al., 1992; Richardson et al., 1992; Bisler et al., 2002; Zangenehpour and Chaudhuri, 2002). The particular time point chosen has allowed distinctions in neuronal activity patterns induced by different radial-arm maze manipulations in previous studies (Maviel et al., 2004). Because memory processes have been revealed to be time dependent and involve distinct cellular populations at different periods (Bontempi et al., 1996; Izquierdo and Medina, 1997; Ambrogi Lorenzini et al., 1999; Igaz et al., 2002), this period is not the only one of interest. Importantly, however, only one peak of zif268 mRNA induction by spatial training was found by Guzowski et al. (2001), which fits the time frame chosen here for Zif268 protein sampling.

After the final test followed by dark room session, the rats were irreversibly anesthetized with sodium pentobarbital $(140 \mathrm{mg} / \mathrm{kg}$, Euthatal; Rhone Merieux, Harlow, UK) and perfused transcardially with $0.1 \mathrm{~m}$ PBS followed by $4 \%$ paraformaldehyde in $0.1 \mathrm{M}$ PBS (PFA). The brains were removed and postfixed in PFA for $4 \mathrm{~h}$ and then transferred to $25 \%$ sucrose overnight. Sections were cut at $40 \mu \mathrm{m}$ on a freezing microtome in the coronal plane. Adjacent series were collected in $0.1 \mathrm{M}$ PBS for Nissl staining and in $0.1 \mathrm{M}$ PBS containing $0.2 \%$ Triton X-100 (PBST) for immunohistochemical visualization of Zif268 protein.

A single experimental cohort of four rats was processed together, sections from each trained rat in the same container as those of its yoked control to minimize immunohistochemical variation (i.e., one RWM-2 rat with one YOKED-2 rat, and one RWM-5 rat with one YOKED-5 rat in a single batch). Endogenous peroxidase activity was blocked by washing the sections with $0.3 \%$ hydrogen peroxide in PBST for $10 \mathrm{~min}$, and then four times with PBST alone for the same duration. Sections were incubated at $4^{\circ} \mathrm{C}$ for $48 \mathrm{~h}$ in PBST with rabbit polyclonal antibody for Zif268 (1:3000, C-19; Santa Cruz Biotechnology, Santa Cruz, CA). Sections were then rinsed for $10 \mathrm{~min}$ in PBST four times. Next they were incubated in biotinylated secondary antibody and then in avidin-biotinylated horseradish peroxidase complex in PBST (Elite Kit; Vector Laboratories, Orton Southgate, Peterborough, UK). Sections were next rinsed in Tris nonsaline buffer, $\mathrm{pH}$ 7.4. Finally, immunoreactivity was visualized with diaminobenzidine (DAB Substrate Kit; Vector Laboratories) chromogen incubation. Sections were then mounted on gelatinized slides. These slides and another series of cresyl violet-stained tissue were dehydrated through a series of alcohol gradients and coverslipped.

\section{Cell counts}

Sections were scanned using a Leitz Dialux 20 microscope equipped with a Dage MTI CCD72S camera interfaced to a PC computer by a Scion (Frederick, MD) LG-3 frame-grabber board. After image processing, counts of the stained nuclei were obtained using the public domain NIH Image program. Counts were made of immunopositive cells that were above a specific threshold from background labeling, a threshold that was the same for all regions. Cortical areas [perirhinal cortex, postrhinal cortex, medial entorhinal cortex (mEnt), and retrosplenial cortex] were assessed (Fig. 2a,b) in a standard frame sample area $(0.78 \times 0.55 \mathrm{~mm})$ that included all laminas, using a $10 \times$ objective. Hippocampal counts were obtained separately for the dentate gyrus, CA3, and CA1 (Fig. 2a,b). The entire extent of the target dorsal hippocampal region is represented by the coronal sections from -2.56 to -3.14 from bregma according to Paxinos and Watson (1997). No separate counts were obtained within the medial entorhinal cortex laminas (II vs III), because no robust boundaries could be drawn to separate Zif268 counts in these laminas. Counts for all regions were typically taken from three consecutive sections from both hemispheres, and these counts were averaged to produce a mean. As stated previously, only the dorsal region of the hippocampus was evaluated (Fig. $2 a$ ), because lesions of the ventral hippocampus need not impair performance of the radial-arm maze concurrent reference and working task (Pothuizen et al., 2004).

\section{Statistical analyses}

Regional Zif268 production. Immediate-early gene cell counts were evaluated by conducting a mixed-design ANOVA on absolute Zif268positive cell counts for the brain regions (repeated measures) in the different testing conditions (between-group measures). Bivariate correlations were calculated using the Pearson product-moment correlation a.
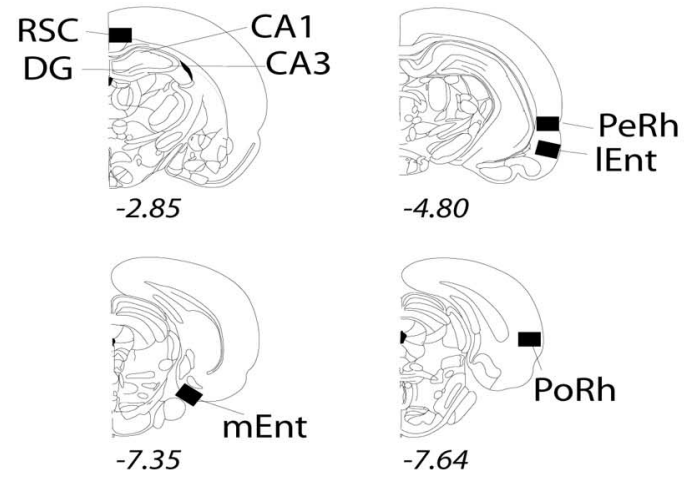

b.

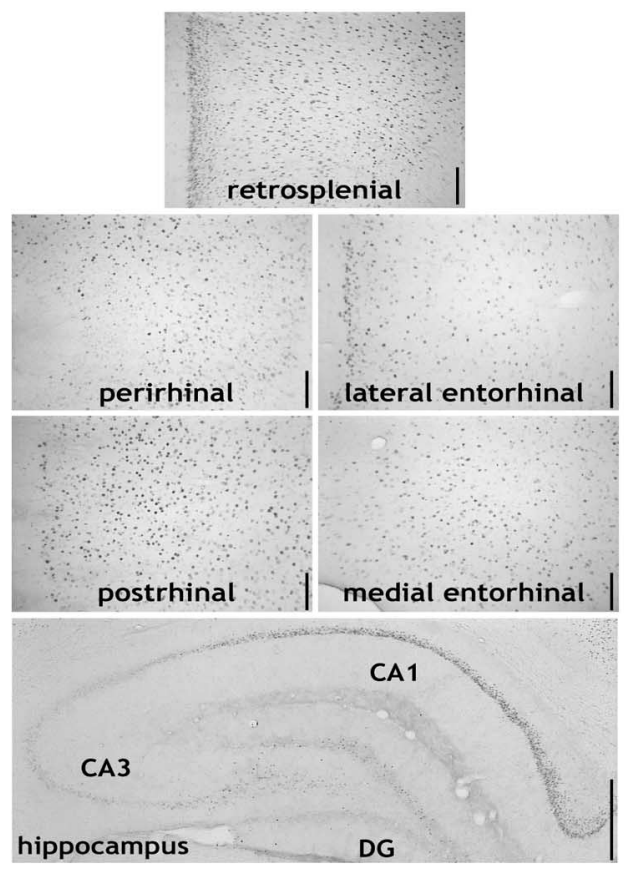

c.

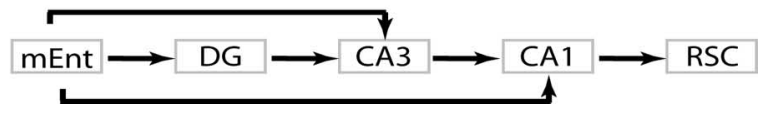

Figure 2. $\quad \boldsymbol{a}$, Coronal sections depicting regions of interest where immediate-early gene cell counts were obtained, with approximate distance from bregma. $\boldsymbol{b}$, Zif268 images from a RWM-5 subject from each region of interest. The superficial laminas are on the left of the cortical slices, except for the medial entorhinal cortex, where the superficial laminas are bottom left. Scale bars: cortical areas, $125 \mu \mathrm{m}$; hippocampus, $500 \mu \mathrm{m}$. C, Pathways used for the anatomically derived models (see text for more information on the path models and the reciprocal connections between the medial entorhinal cortex and the CA1-CA3 fields). PoRh, Postrhinal cortex; RSC, retrosplenial cortex; Perh, perirhinal cortex; IEnt, lateral entorhinal cortex.

coefficient for regional immediate-early gene activity and performance metrics. The SPSS 14.0 (SPSS, Chicago, IL) and JMP 7.0 (SAS Institute, Cary, NC) statistical packages were used for these analyses.

Structural equation modeling. Path analyses were obtained by using structural equation modeling based on the maximum likelihood estimation (MLE), using the statistical package Amos 6.0 (SPSS). It is recommended that when using the MLE method with small samples, the ratio of the $\chi^{2}$ to the degrees of freedom should be $<2$. To remain conservative, in addition to the MLE significance $\chi^{2}$, which may be unreliable with small samples, additional measures of model fit were reported. Unlike the $\chi^{2}$, which provides a dichotomous accept or reject decision, these additional measures offer a degree of fit. It should be noted that path strength remains accurate even in smaller samples (Boucard et al., 2007). 
a. Reference

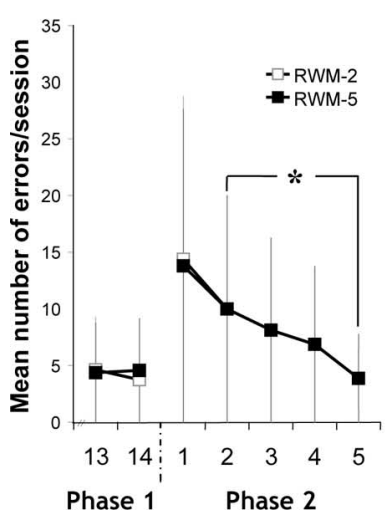

b1. Working (non-reference)

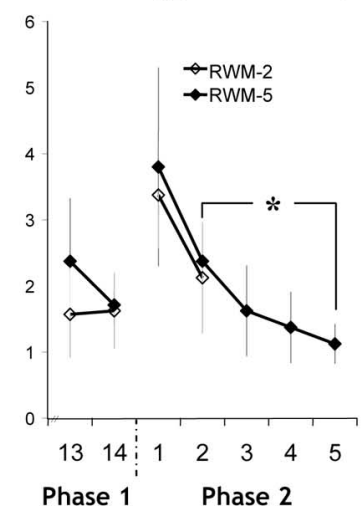

b2. Working (reference)

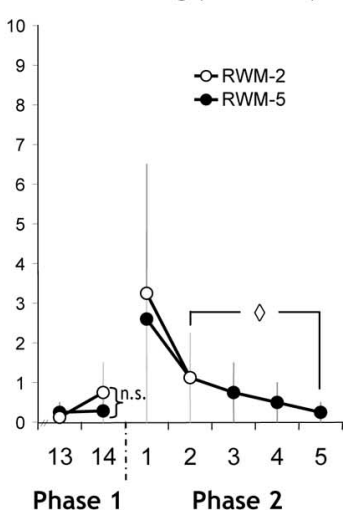

Figure 3. Graphs showing radial-arm maze performance for the last two sessions of phase 1 and all of phase 2 for the two experimental groups (RWM-2, RWM-5). $\boldsymbol{a}$, Mean number of reference memory errors per session. $\boldsymbol{b} 1$, Mean number of working (nonreference) errors per session. $\boldsymbol{b 2}$, Mean number of working (reference) memory errors per session. ${ }^{*} p<0.05$; ${ }^{\diamond} p=0.056$; n.s., $p>0.3$. Error bars represent SEM.

The two additional types of fit indices that were used to determine the robustness of the findings were the goodness-of-fit index (GFI) and the comparative fit index (CFI). The GFI is an index of the proportion of variance accounted. The GFI has a strong "downward bias" with small sample sizes, thus in reality being especially conservative (Fan, 1996), thus addressing a limitation of the current study. Another approach is based on the comparison of those models derived in the current study to independent models, in which no regions are connected. Such independent models have the least fit, and a high index value means that the tested model is opposite to the independent model, thus exhibiting good fit. For this type of comparison, the CFI was chosen because it is a recommended index with small sample sizes (Fan, 1996; Hu and Bentler, 1998). A good-fitting model that is a plausible representation of the underlying structure was considered to either have a GFI $\geq 0.9$ or a CFI $\geq 0.90-0.95$ (Tabachnik and Fidell, 1996). Although this fit threshold is sought, it should be noted that when derived from anatomical connectivity information, Protzner and McIntosh (2006) have argued that differences between conditions are detectable and valid "regardless of absolute model fit." For each dependent brain region in the models, the squared multiple correlation ( $R^{2}$ or coefficient of determination) is also presented; this value represents a measure of the proportion of the variance of the dependent variable that is explained by the independent variable(s).

Estimates of causal inference may be warranted where the connections between the hippocampal subfields are unidirectional, for example those between the medial entorhinal cortex and CA3 (Witter et al., 2000). In the case of the reciprocal projections between the medial entorhinal cortex and CA1 (Witter et al., 2000), paths were tested in both directions. Each model only considered five regions because the number of degrees of freedom precluded an inclusive analysis of all the regions tested (Fig. $2 c)$.

Next, specification searches were conducted, in which anatomically plausible paths between regions were freely estimated using the modification indices to obtain increases in the fit of the model. Thus, final models were anatomically derived (van Groen and Wyss, 1990; Witter et al., 2000; van Groen and Wyss, 2003) and tested against statistical assumptions; they were not simply connectional models that were purely fitted to the data. Finally, a multisample approach completed the path analyses. This approach uses a $\chi^{2}$ difference test, which, although dependent on sample size (differences can be difficult to detect when sample sizes are small), enables the direct comparison of groups.

\section{Results}

\section{Behavioral analyses}

Phase 1 (pretraining)

The experimental groups (RWM-2, RWM-5) behaved similarly in the pretraining phase, on measures of both reference and

working memory. Thus, for all three measures [reference memory errors, working (nonreference) memory errors, working (reference) memory errors] there was a significant effect of session (all $p<$ $0.0001)$, but no difference in the group scores (all $p>0.8$ ). For none of these measures was there a group difference for the last session (Fig. 3); i.e., both groups were equated on pretraining.

\section{Phase 2 (test phase)}

Not every rat visited four separate arms in each of the four trials during the first session, and thus Figure 3 only depicts the mean scores for session 1 for those rats that completed the session. From session 2 onward, all rats completed all trials. To test the validity of the two experimental conditions (RWM-2 and RWM-5 sessions), it was necessary to determine whether groups RWM-2 and RWM-5 were matched on session 2 and whether the five-session group (RWM-5) had continued to improve with subsequent training.

Independent $t$ tests for session 2 scores found no evidence of a performance difference between the RWM-2 and RWM-5 groups at this early stage in task acquisition (Fig. 3) [reference errors, working (nonreference) memory errors, and working (reference) memory errors, all $p>0.7]$. Although early learning may particularly tax extramaze spatial cue acquisition, visual inspection indicated that working memory errors were quickly reduced (Fig. 3), suggesting that the spatial disambiguation of the arms as well as the temporal disambiguation of the arm entries across trials was soon achieved. The current task had unusually high levels of proactive interference because repeated trials were run within a session, so taxing the temporal separation of arm choices. The decline in working memory errors with training could not solely be linked with an improvement in the temporal separation of spatial information, because there was no evidence that extended training (RWM-5) most reduced errors when proactive interference should be greatest, i.e., during the later runs within each trial (data not shown). Temporal separation seemed easiest for those arms that were never rewarded, and thus were unambiguous [working (nonreference) vs working (reference) memory errors] (Fig. 3). Most taxing seemed the acquisition of the arm-reward associations, reflected in the slower reduction of reference memory errors (Fig. 3). The RWM rats' ability to only visit baited arms improved with additional sessions; withinsubject $t$ tests comparing performance on sessions 2 and 5 showed that the five-session animals (RWM-5) benefited from the extra training as reflected by their significant decrease in error scores [reference memory, $p<0.01$; working (nonreference) memory, $p<0.05$; marginally significant for working (reference) memory, $p=0.056$.

The total time the subjects spent in the maze on their last test session (session 2 or 5 ) was compared with a univariate ANOVA for the four groups (two experimental, two yoked controls). The mean amount of time spent by each rat in the maze during the final session was similar for each group (SD values in parentheses): RWM-2, 238.4 s (155.4); YOKED-2, 276.0 s (199.6); RWM-5, $191.5 \mathrm{~s}$ (173.3); and YOKED-5, $151.5 \mathrm{~s}$ (70.6). Critically, there was no significant effect of condition [i.e., training or yoked control $(F<1)$ or extent of maze experience, two versus five 


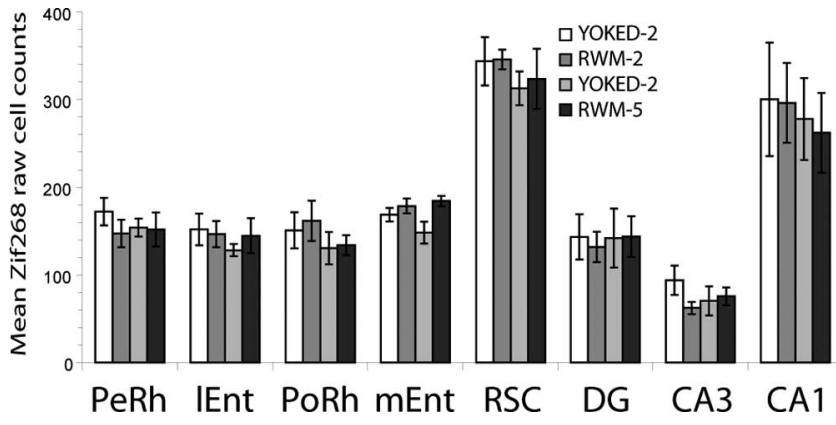

Figure 4. Histogram showing mean and SE of Zif268 cell counts $(n=8)$ for each of the four groups. IEnt, Lateral entorhinal cortex; PeRh, perirhinal cortex; PoRh, postrhinal cortex; RSC, retrosplenial cortex.

sessions $\left.\left(F_{(1,28)}=2.37 ; p=0.14\right)\right]$ on total time spent in the new maze. Additionally, independent-samples $t$ test (two-tailed) confirmed that the total time spent in the second maze (across all training sessions) did not differ between the experimental and yoked subjects for either the two-session or five-session groups (RWM-2 vs YOKED-2 and RWM-5 vs YOKED-5, both $p>0.6$ ).

\section{Immunohistochemical results}

ANOVAs

A mixed-design ANOVA of the absolute Zif268-positive cell counts (Fig. 4) was conducted. The ANOVA included experimental condition (two levels, RWM vs YOKED) and training condition (two sessions or five sessions) as between-subjects factors, and brain region condition (five levels) as a within-subjects factor. This analysis revealed no effect of experimental condition $(F<1)$ or training extent $(F<1)$. No interaction was found between experimental condition and training extent $(F<1)$, experimental condition and brain region $(F<1)$, or brain region and training extent $(F<1)$. Likewise, the three-way interaction between experimental condition, training extent, and brain region was not significant $(F<1)$.

\section{Correlational analyses for brain regions and behavior}

A key question was whether Zif268 counts in CA1, CA3, and the dentate gyrus would differentially correlate with early and late training. For this reason, Table 1 provides the separate correlations between performance indices and Zif268 activity in these sites for "early" (two-session) and "late" (five-session) training.

Significant, positive associations between hippocampal subfield Zif268 activity and performance parameters were found (Table 1, Fig. 5). Six performance scores showed high, positive correlations with dentate gyrus Zif268 levels after early training (RWM-2), three of which were significant [working (nonreference) memory errors in the final session, the degree of improvement from session 1 to 2 for working (reference) memory errors in the final session, and reference memory errors (Table 1)]. In all cases, higher expression of Zif268 protein in the dentate gyrus was associated with poorer performance. Zif268 induction in RWM-2 was also significantly correlated with the total time spent in the maze but only in the dentate gyrus $(r=0.72 ; n=8 ; p<$ $0.05)$; no such correlation was observed for the YOKED-2 group or other regions (supplemental Table S1, available at www. jneurosci.org as supplemental material). For later training (RWM-5), most of these same correlations with dentate gyrus became negative and none were significant.

A very different pattern was observed for the Zif268 counts in the CA3 and CA1 fields. No significant performance correlations
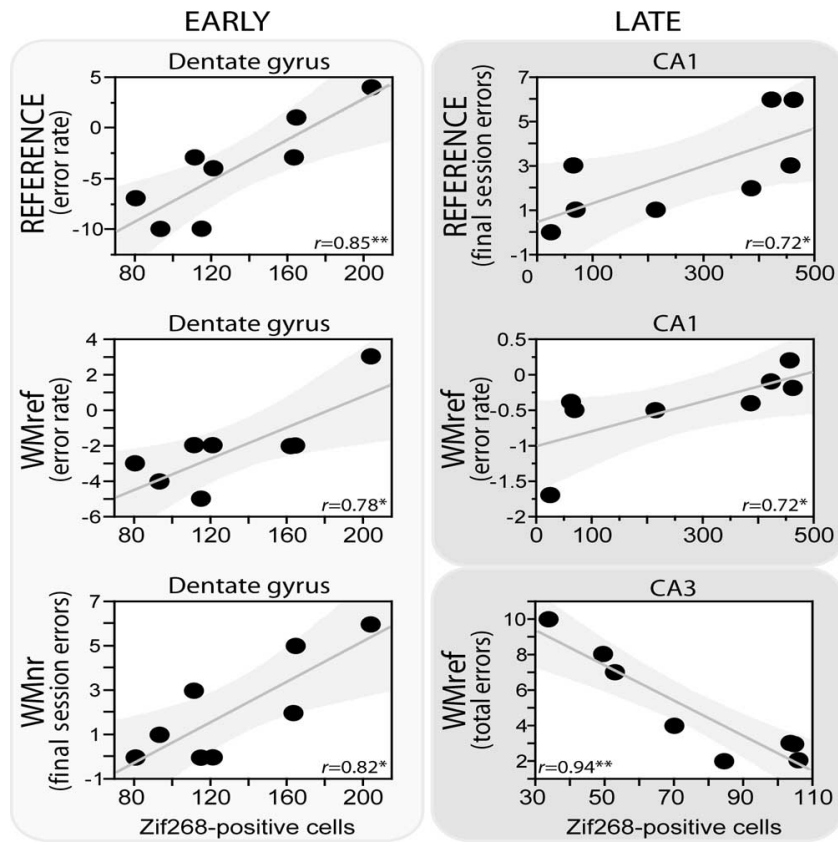

Figure 5. Scatter plots depicting the dissociation in significant correlations between Zif268positive cell counts in hippocampal subfields and performance errors. Notice the dynamic pattern as a switch occurs from the dentate gyrus in early (left, RWM-2) training to CA1 and CA3 subfields with additional training (right, RWM-5). The shaded area around the trend line represents the confidence interval, indicating the strength of the linear relationship. ${ }^{*} p<0.05$; ${ }^{* *} p<0.01$. WMref, Working memory (reference) errors; WMnr, working memory (nonreference) errors.

were found in early learning (RWM-2) (Table 1). In contrast, performance indices in later learning (RWM-5) were now significantly correlated with Zif268 (Table 1). These correlations included repeat visits down never-baited arms [working (reference) memory] for both CA3 (total errors) and CA1 (rate), as well as reference memory for CA1 only. The RWM-5 correlation between CA1 Zif268 and total time spent in the maze approached but did not reach significance $(r=0.69 ; n=8 ; p=0.058)$, unlike the YOKED-5 control $(r=0.72 ; n=8$; $p<0.05)$. It should be noted that, similar to the dentate gyrus, higher Zif268 counts in CA1 were always associated with higher error scores, hence, possibly involving both subfields in error correction. This observation contrasts with the association of higher Zif268 counts in CA3 with lower error scores, and thus possibly related to consolidation processes.

Of all the brain regions examined, only the retrosplenial cortex exhibited associations for working or reference memory selectively according to training level. A significant, positive association was observed only with working memory (nonreference errors) early in training $(r=0.75 ; p<0.05)$, but in contrast, with late training only reference memory (total errors) was significantly correlated $(r=0.72 ; p<0.05)$. The pattern of associations of behavioral measure with retrosplenial cortex is also striking in that this region did not mimic the pattern of CA1, despite very high correlations of Zif268 expression between these two areas (Fig. 6), thus suggesting some functional independence.

Overall, several brain regions exhibited significant associations with behavioral scores [compare details of the results for the postrhinal cortex, perirhinal cortex, and lateral entorhinal cortex (supplemental Table S1, available at www.jneurosci.org as supplemental material)]. To mirror the path analyses and focus on the main question about the functions of the temporoammonic 
Table 1. Correlations between Zif268-positive cell counts and behavioral learning measures in phase 2 for the early (RWM-2) and late (RWM-5) training groups

\begin{tabular}{|c|c|c|c|c|c|c|c|c|c|}
\hline \multirow{7}{*}{ 党 } & Region & Measure & $\begin{array}{c}\text { WMnr } \\
\text { (fs) }\end{array}$ & $\begin{array}{l}\text { WMnr } \\
\text { (rate) }\end{array}$ & $\begin{array}{c}\text { WMref } \\
\text { (fs) }\end{array}$ & $\begin{array}{c}\text { WMref } \\
\text { (t) }\end{array}$ & $\begin{array}{l}\text { WIMref } \\
\text { (rate) }\end{array}$ & $\begin{array}{l}\text { REF } \\
\text { (fs) }\end{array}$ & $\begin{array}{c}\text { REF } \\
\text { (rate) }\end{array}$ \\
\hline & \multirow{2}{*}{ DG } & $r$ & $.816^{\star}$ & .655 & .622 & .331 & .782* & .567 & .854** \\
\hline & & $p$ & .013 & .078 & .100 & .423 & .022 & .142 & .007 \\
\hline & \multirow{2}{*}{ CA3 } & $r$ & -.221 & -.507 & .075 & -.063 & .196 & -.039 & .100 \\
\hline & & $p$ & .598 & .199 & .860 & .882 & .642 & .927 & .815 \\
\hline & \multirow{2}{*}{ CA1 } & $r$ & .506 & .561 & .320 & .256 & .318 & .454 & .418 \\
\hline & & $p$ & .200 & .148 & .439 & .541 & .442 & .259 & .303 \\
\hline \multirow{7}{*}{ 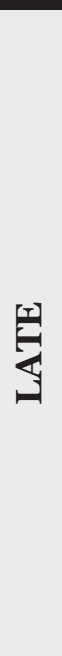 } & Region & Measure & $\begin{array}{c}\text { WMnr } \\
\text { (fs) }\end{array}$ & $\begin{array}{l}\text { WMnr } \\
\text { (rate) }\end{array}$ & $\begin{array}{c}\text { WIMref } \\
\text { (fs) }\end{array}$ & $\begin{array}{c}\text { WIMref } \\
\text { (t) }\end{array}$ & $\begin{array}{l}\text { WIMref } \\
\text { (rate) }\end{array}$ & $\begin{array}{l}\text { REF } \\
\text { (fs) }\end{array}$ & $\begin{array}{c}\text { REF } \\
\text { (rate) }\end{array}$ \\
\hline & \multirow{2}{*}{ DG } & $r$ & -.341 & -.281 & -.327 & .147 & -.056 & .020 & -.286 \\
\hline & & $p$ & .409 & .500 & .429 & .728 & .895 & .963 & .492 \\
\hline & \multirow{2}{*}{ CA3 } & $r$ & .085 & .311 & -.307 & $-.939^{\star *}$ & .328 & -.205 & -.009 \\
\hline & & $p$ & .841 & .453 & .460 & .001 & .427 & .626 & .983 \\
\hline & \multirow{2}{*}{ CA1 } & $r$ & .253 & .300 & .572 & .069 & $.716^{*}$ & $.722^{\star}$ & .564 \\
\hline & & $p$ & .546 & .470 & .138 & .870 & .046 & .043 & .145 \\
\hline
\end{tabular}

WMref, Working memory (reference) errors; WMnr, working memory (non-reference) errors; fs, final session; REF, reference memory errors; rate, across-session slope of improvement. Correlations (two-tailed) significant at the 0.05 (*) or $\left.0.01{ }^{* *}\right)$ level are shaded. $r$, Pearson product-moment coefficient. $n=8$.

versus trisynaptic routes to CA1, emphasis in the discussion will be placed on those regions that form these circuits.

\section{Structural equation modeling}

Although there were no group differences for Zif268 counts, this null result does not mean that the relationships between sites within the same animal may not have changed with experience. It should be reemphasized that the models tested had to be anatomically plausible and that the paths reflect known direct links between the regions.

The correlation matrices for regional Zif268 activity for each behavioral group are summarized in Figure 6. It is important to note that the stronger the correlations between the brain regions, the more power structural equation modeling has to test a model, and a rejection of the null hypothesis refers to an unacceptable model (Tabachnik and Fidell, 1996). Very high interregional brain correlations (Fig. 6) suggested some multicollinearity between the investigated regions. For example, although a stronger indirect route through the subiculum connects the retrosplenial cortex and CA1, these two areas exhibited strong, significant correlations for each group, even approaching singularity. Multicollinearity, while bearing no impact on the predictive value of gen- erated models and their paths, may affect the value of these paths. Where collinearity symptoms appeared (RWM-2, as suggested by squared multiple correlations or path coefficients $>1.00$ ), a model is presented in the supplemental material with collinearity regressed out, leaving no out-of-range values (supplemental Fig. $\mathrm{S} 1$, available at www.jneurosci.org as supplemental material).

\section{Path analyses}

The structural equation modeling analyses provide quantitative estimates of neural coupling associated with different spatial information processing. For the trained subjects, the influences of the medial entorhinal cortex on CA3 and of CA3 on CA1 were significantly reduced with prolonged training (Fig. 7), so distinguishing the putative networks for the early and late training groups. The distinctions between the four groups were determined by first testing a "baseline" model for all the groups, adapting this model where possible, and finally directly comparing the groups by using stacked models to evaluate specific path strength differences. A baseline model (Fig. 2c) including all connections of the trisynaptic circuit and of the temporoammonic paths was used for the pooled data from all animals $(n=32)$. This baseline model did not fit well, as especially determined by the $\chi^{2}$ value 


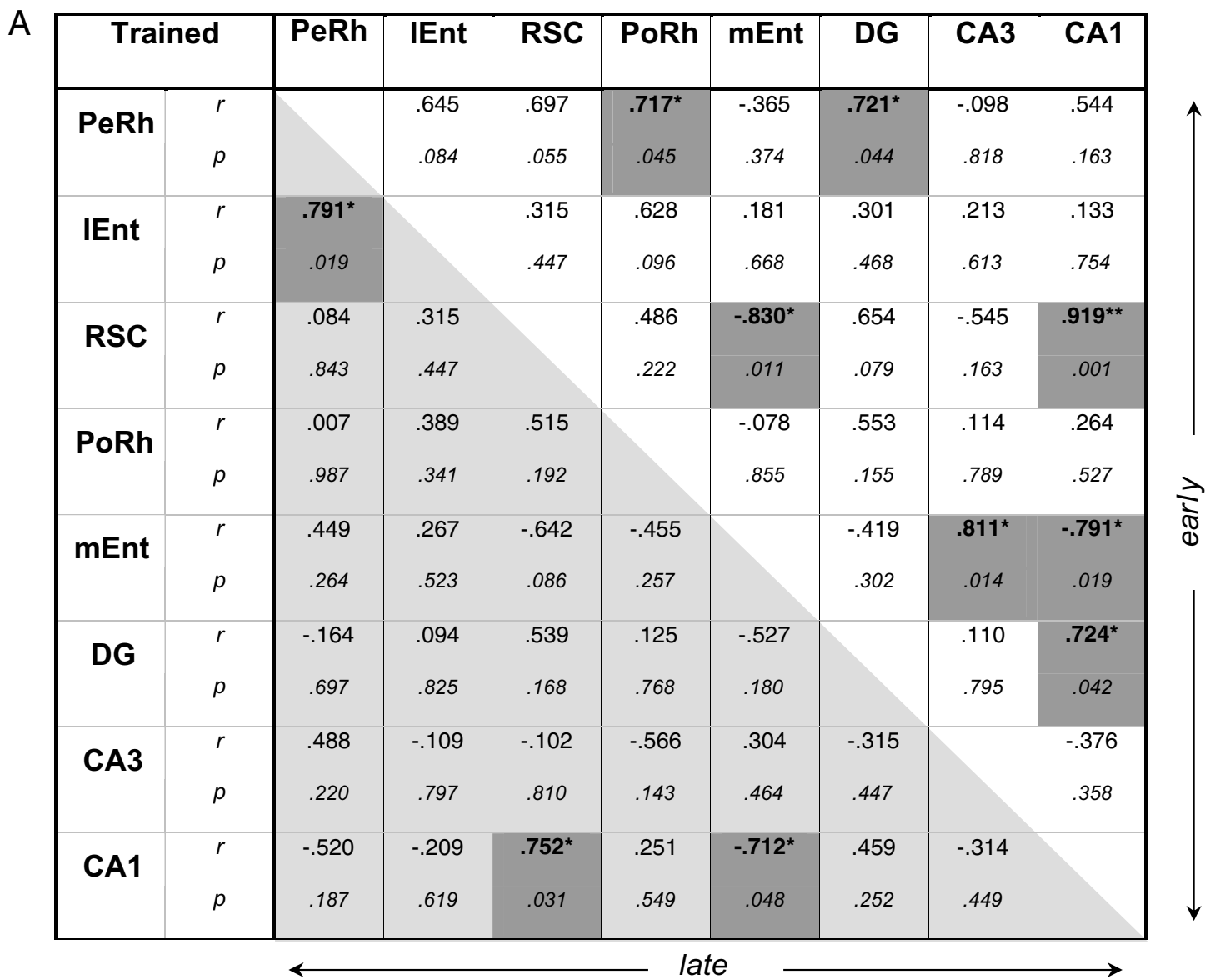

\begin{tabular}{|c|c|c|c|c|c|c|c|c|c|}
\hline \multicolumn{2}{|c|}{ Yoked } & \multirow[t]{3}{*}{ PeRh } & \multirow{2}{*}{$\begin{array}{c}\text { IEnt } \\
.289\end{array}$} & \multirow{2}{*}{$\begin{array}{c}\text { RSC } \\
.025\end{array}$} & \multirow{2}{*}{$\begin{array}{c}\text { PoRh } \\
.306\end{array}$} & \multirow{2}{*}{$\begin{array}{c}\text { mEnt } \\
-.146\end{array}$} & \multirow{2}{*}{$\begin{array}{c}\text { DG } \\
.352\end{array}$} & \multirow{2}{*}{$\begin{array}{c}\text { CA3 } \\
.225\end{array}$} & \multirow{2}{*}{$\begin{array}{l}\text { CA1 } \\
-.024\end{array}$} \\
\hline & $r$ & & & & & & & & \\
\hline & $p$ & & .487 & .953 & .461 & .730 & .393 & .592 & .955 \\
\hline \multirow{2}{*}{ IEnt } & $r$ & .109 & & .423 & .516 & -.079 & .462 & .004 & .267 \\
\hline & $p$ & .797 & & .297 & .190 & .852 & .250 & .992 & .523 \\
\hline \multirow{2}{*}{ RSC } & $r$ & -.457 & .396 & & .643 & -.696 & $.807^{\star}$ & .187 & $.791^{*}$ \\
\hline & $p$ & .255 & .332 & & .085 & .055 & .015 & .657 & .020 \\
\hline \multirow{2}{*}{ PoRh } & $r$ & .295 & .349 & .423 & & -.479 & $.868^{\star \star}$ & .628 & .325 \\
\hline & $p$ & .478 & .396 & .296 & & .229 & .005 & .096 & .432 \\
\hline \multirow{2}{*}{ mEnt } & $r$ & .647 & .204 & $-.729^{*}$ & .006 & & -.491 & -.053 & -.689 \\
\hline & $p$ & .083 & .628 & .040 & .989 & & .217 & .901 & .058 \\
\hline \multirow{2}{*}{ DG } & $r$ & -.425 & .222 & .649 & -.333 & -.597 & & .649 & .360 \\
\hline & $p$ & .294 & .597 & .082 & .420 & .118 & & .082 & .380 \\
\hline \multirow{2}{*}{ CA3 } & $r$ & .096 & .282 & .386 & .010 & -.065 & .609 & & -.317 \\
\hline & $p$ & .820 & .498 & .345 & .981 & .879 & .109 & & .444 \\
\hline \multirow{2}{*}{ CA1 } & $r$ & -.649 & .242 & $.899^{\star \star}$ & .038 & $-.847^{\star \star}$ & $.804^{\star}$ & .229 & \\
\hline & $p$ & .082 & .563 & .002 & .929 & .008 & .016 & .585 & \\
\hline
\end{tabular}




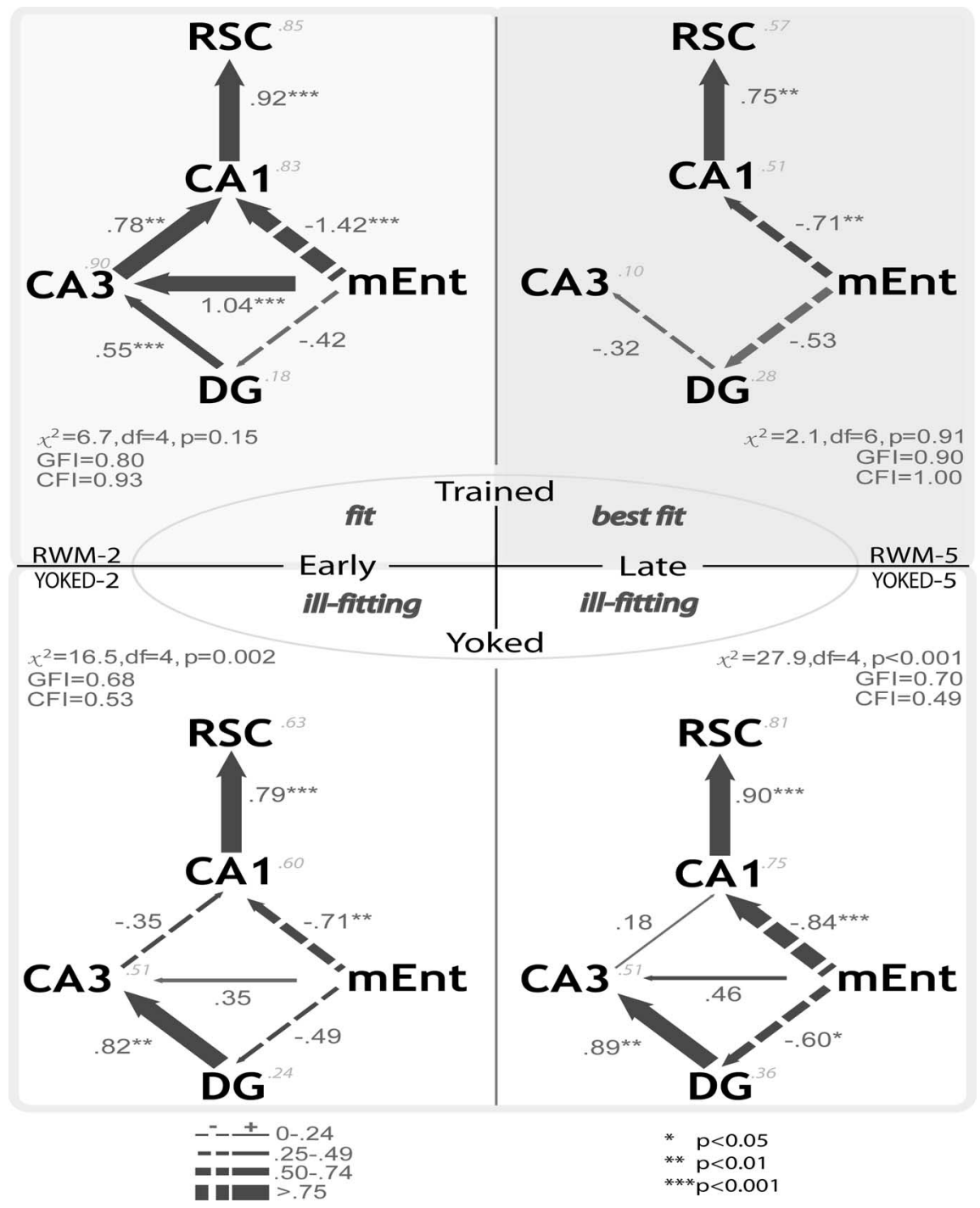

Figure 7. Path analyses for the rats trained (top two) in the radial-arm maze for either two (RWM-2, "early") or five (RWM-5, "late") sessions. The bottom two path analyses are for their respective yoked controls (YOKED-2, YOKED-5). ${ }^{*} p<0.05$; ${ }^{* *} p<$ $0.01 ;{ }^{* * *} p<0.001$. Notice the preponderance of the temporoammonic path to CA1 over the trisynaptic circuit induced with training. The strength and the positive or negative relation of the causal influences are depicted in the diagrams and explained in the figure. The squared multiple correlations $\left(R^{2}\right)$ are in italics. RSC, Retrosplenial cortex.

$\left(\chi^{2}=13.54 ; \mathrm{df}=4 ; p=0.009 ; \mathrm{GFI}=0.87 ; \mathrm{CFI}=0.88\right)$. The results of this analysis emphasized that the underlying, principal anatomical connectivity did not necessarily translate into strong effective connectivity.

This baseline model was subsequently used as a starting point to individually fit models for group. This baseline model yielded a high fit for the late training group (RWM-5), but the weakness of certain paths enabled a simplification of the model to a more parsimonious version, while still maintaining this high level of fit $\left(\chi^{2}=2.14 ; \mathrm{df}=6 ; p=0.91 ; \mathrm{GFI}=0.90 ; \mathrm{CFI}=1.00\right)$ (Fig. 7 , top right). In contrast, the early training group (RWM-2) required all existing paths to even manage a good but relatively lower fit $\left(\chi^{2}\right.$ $=6.7 ; \mathrm{df}=4 ; p=0.15 ; \mathrm{GFI}=0.80 ; \mathrm{CFI}=0.93)$. However, not only was this model fit acceptable for the early training group, but the proportion of variance explained was high, as revealed by the $R^{2}$ values, lending especially good predictive value of the model in this condition. It should be noted that substituting the lateral entorhinal cortex for the medial entorhinal cortex yielded a severely deteriorated fit for both groups (data not shown).

Strikingly, it remained impossible to generate a good-fitting model for either YOKED group. It should be noted that the model for the early training group suffers from multicollinearity, as suggested by the out-of-range, symptomatic path coefficients (Fig. 7, RWM-2). Although all groups exhibit very high interregional correlations between the retrosplenial cortex and CA1, the early training group exhibited the highest such correlation, in addition to several others. The high level of apparent cohesion in this group is likely the cause of the statistical collinearity, which nonetheless does not diminish the predictive value of the results.

Because different fitting models were obtained for each trained group, and none for the yoked groups, the lack of a common fitting common model for all four groups precludes a direct comparison of their paths. This observation emphasizes the different dynamics for these groups and especially for the yoked groups. Inspection of the optimal models for each training group (Fig. 7) suggested that several path estimates were different between the two levels of training. These paths included those of the trisynaptic loop $[\mathrm{mEnt} \rightarrow \mathrm{DG}$, $\mathrm{DG} \rightarrow \mathrm{CA} 3, \mathrm{CA} 3 \rightarrow \mathrm{CA} 1]$, as well as that from the medial entorhinal cortex to CA3, but not that from the medial entorhinal cortex to CA1, nor from CA1 to the retrosplenial cortex. This observation was supported by the discrepancies between the groups in the squared multiple correlation coefficients, which indicated that the relations between the variables changed widely according to the condition (see especially CA3 in Fig. 7).

To compare formally the path coefficients between the two training levels, we used a multisample approach. Here the two groups were stacked together, using a model that included all the paths to be tested. For each analysis, the model was first estimated with none of the parameters constrained and then compared with a more restricted model with parameters constrained across the groups.

The unconstrained stacked model $\left(\chi^{2}=18.03 ; \mathrm{df}=13 ; p=\right.$ 0.16 ; GFI $=0.73$; $\mathrm{CFI}=0.82)$ exhibited a reduced fit relative to

$\leftarrow$

Figure 6. Interregion correlations of Zif268 counts from the two experimental groups (RWM-2, RWM-5; $\boldsymbol{A}$ ) and from their respective yoked controls (YOKED-2, YOKED-5; $\boldsymbol{B}$ ). The top right diagonal matrix concerns data from the Early session ( $\boldsymbol{A}, \mathrm{RWM}-2 ; \boldsymbol{B}$, YOKED-2); the bottom left diagonal matrix concerns data from the Late session $(\boldsymbol{A}, \mathrm{RWM}-5 ; \boldsymbol{B}$, YOKED-5), $\boldsymbol{r}$, Pearson product-moment coefficient; IEnt, lateral entorhinal cortex; PeRh, perirhinal cortex; PoRh, postrhinal cortex; RSC, retrosplenial cortex. Correlations (two-tailed) that are significant at the $0.05\left({ }^{*}\right)$ or 0.01 level $\left({ }^{* *}\right)$ are shaded. $n=8$ per group. 
Table 2. Results of the multiple-sample structural equation model analyses in which stacked early and late trained groups were evaluated with specific path constraints

\begin{tabular}{|c|c|c|c|c|c|c|c|c|}
\hline \multirow[b]{2}{*}{ Stacked model constraints } & \multicolumn{5}{|c|}{ Model fit } & \multicolumn{3}{|c|}{ Model comparisons (to unconstrained) } \\
\hline & $\chi^{2}$ & df & $p$ & $\mathrm{GFI}$ & $\mathrm{CFI}$ & df & $\Delta \chi^{2}$ & $p$ \\
\hline Unconstrained & 18.04 & 13 & 0.16 & 0.73 & 0.82 & & & \\
\hline All paths & 22.08 & 19 & 0.08 & 0.68 & 0.67 & 6 & 10.04 & 0.123 \\
\hline $\mathrm{mEnt}-\mathrm{CA} 1^{a}$ & 22.73 & 14 & 0.07 & 0.70 & 0.69 & 1 & 4.68 & $0.030^{*}$ \\
\hline $\mathrm{mEnt}-\mathrm{CA} 1^{b}$ & 18.97 & 14 & 0.17 & 0.72 & 0.75 & 1 & 0.93 & 0.335 \\
\hline mEnt-CA3 & 22.40 & 14 & 0.07 & 0.74 & 0.70 & 1 & 4.35 & $0.037^{*}$ \\
\hline CA3-CA1 & 22.10 & 14 & 0.08 & 0.70 & 0.71 & 1 & 4.05 & $0.044^{*}$ \\
\hline DG-CA3 & 21.06 & 14 & 0.10 & 0.73 & 0.75 & 1 & 3.02 & 0.082 \\
\hline mEnt to DG & 18.07 & 14 & 0.20 & 0.73 & 0.85 & 1 & 0.03 & 0.865 \\
\hline CA1-RSC & 18.07 & 14 & 0.20 & 0.73 & 0.85 & 1 & 0.02 & 0.883 \\
\hline mEnt-CA3-CA1 & 26.45 & 15 & $0.03^{*}$ & 0.70 & 0.59 & 2 & 8.41 & $0.015^{*}$ \\
\hline
\end{tabular}

This approach formally demonstrated a selective difference between the two spatial task mastery levels in training, which was restricted to the mEnt $\rightarrow$ CA3 and the CA3 $\rightarrow$ CA1 connections. df, Degrees of freedom; RSC, retrospenial cortex. Statistical significance at the 0.05 level $(*)$ is indicated in bold.

${ }^{a}$ An out-of-range path coefficient for RWM-2 (also seen in Fig. 7) artificially inflated the difference between the two groups).

${ }^{b}$ In order to directly compare the path strength from the medial entorhinal cortex to CA1 between the two training levels in the absence of multicollinearity issues, the variance explained by the medial entorhinal cortex was regressed out of $\mathrm{CA}$, resulting in a $\mathrm{mEnt} \rightarrow \mathrm{CA} 1$ path coefficient that was within range. Constraining this path then no longer yielded a between-group difference.

the individual models (see above and Fig. 7) and to the model of the pooled trained subjects $\left(\chi^{2}=3.98 ; \mathrm{df}=4 ; p=0.41 ; \mathrm{GFI}=\right.$ 0.92 ; CFI $=1.00)$. The poorer fit likely reflects the uneven structure of the data for the two RWM groups. Examination of the result of the $\chi^{2}$ difference test between the unconstrained stacked model and another model in which all paths were simultaneously constrained revealed that the overall structure of the two groups (RWM-2 and RWM-5) was, however, similar $\left(\Delta \chi^{2}=10.04\right.$ with $6 \mathrm{df} ; p=0.12)$. This lack of difference in the overall network structure of the early and late trained groups effectively replicated the baseline path model in two independent samples, thus suggesting that our findings are not the result of overfitting a model to a specific sample. To follow up the suggested selective differences in the networks, the relationship between specific regions was next tested by only constraining individual paths. This approach enabled us to address directly the main hypothesis: that hippocampal system interregion activity may be selectively dynamic, despite similar overall absolute levels of regional Zif268 activity between test conditions. Table 2 presents the results of the analyses of specific path constraints.

Only the paths from the medial entorhinal cortex to CA3 and thence to CA1 exhibited a difference in strength between the two training levels. When both of these paths were constrained simultaneously, the $\chi^{2}$ difference test revealed a deterioration of the model (see last row of Table 2), indicating different path strength across training levels in $\mathrm{mEnt} \rightarrow \mathrm{CA} 3 \rightarrow \mathrm{CA} 1$. The location of this connection-specific effect of task mastery is represented in Figure 7. For the trained subjects, it thus emerged that the initial influence of the medial entorhinal cortex on CA3 and of CA3 on CA1 was significantly reduced with prolonged training (Fig. 7). This specific difference in regional interaction distinguished the putative networks for the early and late training groups, because the reduction of the influence of the dentate gyrus on CA3 did not attain statistical significance.

Importantly, changing the causal structure by reversing the direction of all the paths yielded poorer results (data not shown). This finding suggests that the causal structure presented here may be more consistent with the data. Modeling the bidirectional medial entorhinal cortex and CA1 connections individually for each direction allowed us to verify the possibility that the balance of communication between these regions may switch in parallel with the balance of encoding versus retrieval (Hasselmo, 2005). Specifically representing the return projection from CA1 to the medial entorhinal cortex yielded a poorer model for the early training group (RWM-2); however, it yielded a statistically equiv- alent models for the late training group (RWM-5). This observation allows for the possibility that causality between these two regions may be equivalent in the late training group. Models that instead included covariance of the medial entorhinal cortex and CA1 yielded a nonsignificant relationship between these two regions in all four groups (RWM-2, YOKED-2, RWM-5, and YOKED-5; data not shown). This observation is in contrast to the significant results for the path coefficients that were obtained by modeling unidirectional connections only, so possibly suggesting that a biased balance of influence between the reciprocally connected medial entorhinal cortex and CA1 may better explain the current data.

It should be noted that in interpreting structural modeling results based on 2-deoxyglucose data, it has been argued that negative path coefficients need not necessarily be regarded as the reflection of inhibitory influences (McIntosh and GonzalezLima, 1991), a view also valid for immediate-early gene imaging. It was suggested that these coefficients should rather be understood in the same manner as correlation coefficients, such that a negative coefficient would mean that each unit increase in a region would result in a proportional decrease in the region(s) to which it is connected (McIntosh and Gonzalez-Lima, 1991).

\section{Discussion}

Using Zif268 expression as an indirect measure of plasticity, clear evidence was found of a switch from dentate gyrus-correlated activity in early (two-session) training to CA1- and CA3correlated activity with further training (five sessions) of a task requiring spatial and temporal memory. Higher Zif268 induction in the dentate gyrus was associated with poorer, early performance. Strikingly, when both CA1 and CA3 became associated with performance later in training, this relationship was opposite in each field: higher Zif268 expression was associated with poorer performance in CA1 but with better performance in CA3. This training-induced pattern was reflected in derived hippocampal network dynamics revealed by structural equation modeling, because with additional training, (1) there was a statistical loss of dentate gyrus efferents, and (2) uncoupling of CA3 and CA1 with additional training accompanied their emerging, opposite relations with behavior.

Strikingly, the well fitting network models for the trained subjects failed with the yoked subjects. Thus, although the trained and yoked groups spent comparable times in the test environment and exhibited similar regional Zif268 activity, they seemingly displayed different effective connectivity patterns. This re- 
sult has important repercussions for the interpretation of data based solely on independent levels of neural activity measures. The fact that changes in effective connectivity can be masked in this way has parallels in human imaging studies. Maguire et al. (2000), for example, reported that cortical and hippocampal regions were each activated to equivalent levels by different memory tasks: each, however, was integrated into different networks, producing a unique "neural context" (McIntosh, 1999).

The trained groups exhibited dynamic network interactivity, as predicted by earlier studies (Bontempi et al., 1996; Ros et al., 2006). Putative Zif268-mediated plasticity underlying successful performance of the radial arm-maze task may be reliant on cohesive hippocampal and extrahippocampal activity in early training, followed by network streamlining with task proficiency (Figs. 6, 7) (Buzsáki and Chrobak, 1995). Spatial goal-directed training (RWM-2 vs RWM-5) may elicit synaptic modifications in certain projections, including $\mathrm{mEnt} \rightarrow \mathrm{CA} 3$ and $\mathrm{CA} 3 \rightarrow \mathrm{CA} 1$, but less so in $\mathrm{mEnt} \rightarrow \mathrm{CA} 1$ or DG $\rightarrow$ CA3 (Treves and Rolls, 1992; Hasselmo et al., 2002; present study). The strength of DG $\rightarrow$ CA3 was not statistically different between the two training levels, whereas that of $\mathrm{mEnt} \rightarrow \mathrm{CA} 1$ seemed equivalent between all four groups, regardless of their experience type (trained vs yoked) or duration (early vs late). Based on the path analyses, we propose that the CA3 afferents (its temporoammonic path and the Schaffer collaterals) become less influential with additional training, leaving CA1 to be coupled with the medial entorhinal cortex directly via the its own temporoammonic pathway, not the trisynaptic circuit. This view is consistent with the proposed importance of temporoammonic but not trisynaptic circuit inputs to CA1 in the maintenance of spatial representations (Brun et al., 2002), and in the consolidation of intermediate- or long- but not short-term memory (Remondes and Schuman, 2004; Vago et al., 2007). Our findings support the view that, via their physiological differences and their parallel inputs from the entorhinal cortex, hippocampal subfields can contribute to spatial tasks in distinct ways (Lörincz and Buzsáki, 2000; Witter et al., 2000; Brun et al., 2002; Kesner et al., 2004).

The present study is not the first to look at hippocampal Zif268 after spatial learning (Guzowski et al., 2001; Bozon et al., 2002, 2003; Maviel et al., 2004) or IEG activity (c-Fos) in relation to radial-arm maze activity (Vann et al., 2000; He et al., 2002a,b), but is unique in certain critical ways. All rats were pretrained in a different room and maze, but with the same rules to increase the likelihood that the final results reflect the acquisition and use of new spatial associations and not general task attributes. The reference memory component is especially relevant given the likely importance of zif268 mRNA and protein expression for longterm spatial learning (Abraham et al., 1993; Jones et al., 2001; Bozon et al., 2002; Davis et al., 2003; Maviel et al., 2004). This involvement was supported by the various correlations with regional activity (Table 1). Strikingly, the association of Zif268 with reference memory was particularly stable for the postrhinal cortex, a region exhibiting spatial correlates, and where lesions produce radial-arm maze reference memory deficits (Liu and Bilkey, 2002; Burwell and Hafeman, 2003). In contrast, retrosplenial cortex correlations switched from working (early) to reference (late) memory. This switch may reflect evidence that this region works as an interface between prefrontal cortex and medial temporal lobe functions (Kobayashi and Amaral, 2003).

In distinction from the correlation analyses, direct comparisons of the numbers of Zif28-positive cells failed to distinguish the four groups. This null result may seem surprising given that mastery of a spatial reference memory task in a water maze (ses- sion 1 vs session 7) reduced zif268 mRNA activity in the dorsal hippocampus (Guzowski et al., 2001). In fact, other studies of spatial learning have failed to see significant hippocampal zif268 changes (Wisden et al., 1990; Richter-Levin et al., 1998; Kubik et al., 2007). Indeed, Guzowski et al. (2001) did not find zif268 mRNA level changes in the dorsal hippocampus between rats trained to swim to a hidden platform or a visible cue in the water maze. Furthermore, comparisons between session 1 and session 7 of the water maze (Guzowski et al., 2001) are problematic because there will be differences in stress and arousal levels, as well as changes in the lengths and locations of swim paths. The present study tried to minimize these factors with pretraining and yoked controls. Moreover, the radial-arm maze was chosen because it limits the animals' movements so that yoked controls provide an exact match for the number and location of arms explored, as well as the rate and location of food rewards. This precise matching, along with the fact that incidental learning could not be blocked (Kubik et al., 2007), presumably explains the lack of any overall Zif268 differences.

The correlational findings fit the general model that the dentate gyrus, and its influence on CA3, is more important for the encoding of spatial information but not necessarily for retrieval processes, whereas CA3 and especially CA1 are more critical for consolidation or retrieval processes (Jarrard et al., 1984; McClelland and Goddard, 1996; Lassalle et al., 2000; Daumas et al., 2005; Rolls and Kesner, 2006; Vago et al., 2007). Although a previous study was able to link zif268 activation in CA1 with context retrieval (Hall et al., 2001), the present study could not test this possibility directly. Nevertheless, later association of CA1 Zif268 with behavior scores in the present study can be matched with proposals that CA1 is involved in reading off previously encoded information (McClelland and Goddard, 1996).

The early ability to discriminate arms and the accompanying dentate gyrus Zif268 pattern fit the putative roles for the dentate gyrus in spatial pattern separation (O'Reilly and McClelland, 1994; McClelland and Goddard, 1996; Rolls, 1996; Lörincz and Buzsáki, 2000; Gilbert et al., 2001), spatial novelty (Lee et al., 2005), and "error" processing (Lörincz and Buzsáki, 2000). Our findings also fit well with the proposed role of the dentate gyrus in driving the establishment of new spatial representations in CA3 (McNaughton and Morris, 1987; Treves and Rolls, 1992), which itself may be accompanied by late, not early changes in CA3 communication (Rekart et al., 2007).

The sequential engagement by Zif268 of the hippocampal subfields, first the dentate gyrus and then CA3-1 fields, may be related to their functional differences. For example, the trainingrelated switch may be associated with the superior ability of the dentate gyrus over CA3 to disambiguate spatial environments (Leutgeb et al., 2007). Likewise, the dentate gyrus processes coarse, landmark information, whereas more refined, detailed processing of spatial features occurs in CA1 (Jacobs and Schenk, 2003; Kemp and Manahan-Vaughan, 2007).

CA3 and CA1 Zif268 became linearly uncoupled with further learning, as an opposite relation emerged between their respective Zif268 expression and behavior. CA3-CA1 uncoupling accords with electrophysiological data, as new environment remapping arises independently in CA3 and CA1 (Leutgeb et al., 2004, 2005). This pattern could reflect training-related modulation of CA3 inhibition via the entorhinal cortex and the septum (Swanson et al., 1981; Jones, 1993).

Remapping occurs quickly (Wilson and McNaughton, 1993; Leutgeb et al., 2004), yet the late engagement of fields CA3-CA1 by Zif268 expression only emerged after several sessions in the 
new maze. This finding weakens the plausibility of a role for Zif268 in the initial processing of novel spatial information by CA1 (Jarrard, 1978; Naber et al., 2000). Importantly, however, such delayed induction of Zif268 may be related to maintenance processes, because this protein is associated with stabilization but not generation of CA1 place cell fields in a new environment (Renaudineau et al., 2007). Moreover, familiarity elevates CA1 interneuron firing relative to novelty (Wilson and McNaughton, 1993; Fyhn et al., 2002), opposite to the dentate gyrus pattern (Nitz and McNaughton, 2004).

Our results emphasize that similar overall neural activity levels do not necessarily reflect similar network communication, and that a single snapshot of relative subfield activity taken during acquisition will lack generality. Our findings not only provided striking evidence of training-related switching in the contributions of hippocampal subfields and other connected regions on this spatial memory task, but also help to narrow down those models of how hippocampal subfields interact to support memory.

\section{References}

Abraham WC, Mason SE, Demmer J, Williams JM, Richardson CL, Tate WP, Lawlor PA, Dragunow M (1993) Correlations between immediate early gene induction and the persistence of long-term potentiation. Neuroscience $56: 717-727$.

Abraham WC, Christie B, Logan B, Lawlor P, Dragunow M (1994) Immediate early gene expression associated with the persistence of heterosynaptic long-term depression in the hippocampus. Proc Natl Acad Sci USA 91:10049-10053.

Ambrogi Lorenzini CG, Baldi E, Bucherelli C, Sacchetti B, Tassoni G (1999) Neural topography and chronology of memory consolidation: a review of functional inactivation findings. Neurobiol Learn Mem 71:1-18.

Bannerman DM, Good MA, Butcher SP, Ramsay M, Morris RGM (1995) Distinct components of spatial learning revealed by prior training and NMDA receptor blockade. Nature 378:182-186.

Bisler S, Schleicher A, Gass P, Stehle JH, Zilles K, Staiger JF (2002) Expression of c-Fos, ICER, Krox-24 and JunB in the whisker-to-barrel pathway of rats: time course of induction upon whisker stimulation by tactile exploration of an enriched environment. J Chem Neuroanat 23:187-198.

Bontempi B, Jaffard R, Destrade C (1996) Differential temporal evolution of post-training changes in regional brain glucose metabolism induced by repeated spatial discrimination training in mice: visualization of the memory consolidation process? Eur J Neurosci 8:2348-2360.

Boucard A, Marchand A, Nogues X (2007) Reliability and validity of structural equation modeling applied to neuroimaging data: a simulation study. J Neurosci Methods 166:278-292.

Bozon B, Davis S, Laroche S (2002) Regulated transcription of the immediate-early gene Zif268: mechanisms and gene dosage-dependent function in synaptic plasticity and memory formation. Hippocampus 12:570-577.

Bozon B, Davis S, Laroche S (2003) A requirement for the immediate early gene zif268 in reconsolidation of recognition memory after retrieval. Neuron 40:695-701.

Brun VH, Otnass MK, Molden S, Steffenach H-A, Witter MP, Moser M-B, Moser EI (2002) Place cells and place recognition maintained by direct entorhinal-hippocampal circuitry. Science 296:2243-2246.

Burwell RD, Hafeman DM (2003) Positional firing properties of postrhinal cortex neurons. Neuroscience 119:577-588.

Buzsáki G, Chrobak JJ (1995) Temporal structure in spatially organized neuronal ensembles: a role for interneuronal networks. Curr Opin Neurobiol 5:504-510.

Cain DP (1998) Testing the NMDA, long-term potentiation, and cholinergic hypotheses of spatial learning. Neurosci Biobehav Rev 22:181-193.

Daumas S, Halley H, Frances B, Lassalle J-M (2005) Encoding, consolidation, and retrieval of contextual memory: differential involvement of dorsal CA3 and CA1 hippocampal subregions. Learn Mem 12:375-382.

Davis S, Bozon B, Laroche S (2003) How necessary is the activation of the immediate early gene zif268 in synaptic plasticity and learning? Behav Brain Res 142:17-30.
Dragunow M, Faull R (1989) The use of c-fos as a metabolic marker in neuronal pathway tracing. J Neurosci Methods 29:261-265.

Fan X (1996) The effects of sample size, estimation methods and model specification on SEM indices. Paper presented at Annual Meeting of the American Educational Research Association, New York, April.

Friston KJ, Frith CD, Frackowiak RSJ (1993) Time-dependent changes in effective connectivity measured with PET. Hum Brain Mapp 1:69-79.

Fyhn M, Molden S, Hollup S, Moser M-B, Moser EI (2002) Hippocampal neurons responding to first-time dislocation of a target object. Neuron 35:555-566.

Gass P, Herdegen T, Bravo R, Kiessling M (1992) Induction of immediate early gene encoded proteins in the rat hippocampus after bicucullineinduced seizures: differential expression of KROX-24, FOS and JUN proteins. Neuroscience 48:315-324.

Gilbert PE, Kesner RP, Lee I (2001) Dissociating hippocampal subregions: a double dissociation between dentate gyrus and CA1. Hippocampus 11:626-636.

Guzowski JF (2002) Insights into immediate-early gene function in hippocampal memory consolidation using antisense oligonucleotide and fluorescent imaging approaches. Hippocampus 12:86-104.

Guzowski JF, Setlow B, Wagner EK, McGaugh JL (2001) Experiencedependent gene expression in the rat hippocampus after spatial learning: a comparison of the immediate-early genes arc, c-fos, and zif268. J Neurosci 21:5089-5098.

Hall J, Thomas KL, Everitt BJ (2001) Cellular imaging of zif268 expression in the hippocampus and amygdala during contextual and cued fear memory retrieval: selective activation of hippocampal CA1 neurons during the recall of contextual memories. J Neurosci 21:2186-2193.

Hasselmo ME (2005) What is the function of hippocampal theta rhythm?Linking behavioral data to phasic properties of field potential and unit recording data. Hippocampus 15:936-949.

Hasselmo ME, Hay J, Ilyn M, Gorchetchnikov A (2002) Neuromodulation, theta rhythm and rat spatial navigation. Neural Netw 15:689-707.

He J, Yamada K, Nabeshima T (2002a) A role of Fos expression in the CA3 region of the hippocampus in spatial memory formation in rats. Neuropsychopharmacology 26:259-268.

He J, Yamada K, Nakajima A, Kamei H, Nabeshima T (2002b) Learning and memory in two different reward tasks in a radial arm maze in rats. Behav Brain Res 134:139-148.

Herdegen T, Leah JD (1998) Inducible and constitutive transcription factors in the mammalian nervous system: control of gene expression by Jun, Fos and Krox, and CREB/ATF proteins. Brain Res Brain Res Rev 28:370-490.

Hu L, Bentler PM (1998) Fit indices in covariance structure modeling: sensitivity to underparameterized model misspecification. Psychol Methods 3:424-453.

Hughes PE, Alexi T, Walton M, Williams CE, Dragunow M, Clark RG, Gluckman PD (1999) Activity and injury-dependent expression of inducible transcription factors, growth factors and apoptosis-related genes within the central nervous system. Prog Neurobiol 57:421-450.

Igaz LM, Vianna MR, Medina JH, Izquierdo I (2002) Two time periods of hippocampal mRNA synthesis are required for memory consolidation of fear-motivated learning. J Neurosci 22:6781-6789.

Izquierdo I, Medina JH (1997) Memory formation: the sequence of biochemical events in the hippocampus and its connection to activity in other brain structures. Neurobiol Learn Mem 68:285-316.

Jacobs LF, Schenk F (2003) Unpacking the cognitive map: the parallel map theory of hippocampal function. Psychol Rev 110:285-315.

Jarrard L (1978) Selective hippocampal lesions: differential effects on performance by rats of a spatial task with preoperative versus postoperative training. J Comp Physiol Psychol 92:1119-1127.

Jarrard LE, Okaichi H, Steward O, Goldschmidt RB (1984) On the role of hippocampal connections in the performance of place and cue tasks: comparisons with damage to hippocampus. Behav Neurosci 98:946-954.

Jenkins TA, Amin E, Harold GT, Pearce JM, Aggleton JP (2003) Distinct patterns of hippocampal formation activity associated with different spatial tasks: a Fos imaging study in rats. Exp Brain Res 151:514-523.

Jones MW, Errington ML, French PJ, Fine A, Bliss TV, Garel S, Charnay P, Bozon B, Laroche S, Davis S (2001) A requirement for the immediate early gene Zif268 in the expression of late LTP and long-term memories. Nat Neurosci 4:289-296. 
Jones RSG (1993) Entorhinal-hippocampal connections: a speculative view of their function. Trends Neurosci 16:58-64.

Kemp A, Manahan-Vaughan D (2007) Hippocampal long-term depression: master or minion in declarative memory processes? Trends Neurosci 30:111-118

Kesner RP, Lee I, Gilbert P (2004) A behavioral assessment of hippocampal function based on a subregional analysis. Rev Neurosci 15:333-351.

Kobayashi Y, Amaral DG (2003) Macaque monkey retrosplenial cortex: II. Cortical afferents. J Comp Neurol 466:48-79.

Kubik S, Miyashita T, Guzowski JF (2007) Using immediate-early genes to map hippocampal subregional functions. Learn Mem 14:758-770.

Lassalle J-M, Bataille T, Halley H (2000) Reversible inactivation of the hippocampal mossy fiber synapses in mice impairs spatial learning, but neither consolidation nor memory retrieval, in the Morris navigation task. Neurobiol Learn Mem 73:243-257.

Lee I, Kesner RP (2004) Encoding versus retrieval of spatial memory: double dissociation between the dentate gyrus and the perforant path inputs into CA3 in the dorsal hippocampus. Hippocampus 14:66-76.

Lee I, Hunsaker MR, Kesner RP (2005) The role of hippocampal subregions in detecting spatial novelty. Behav Neurosci 119:145-153.

Leutgeb JK, Leutgeb S, Moser M-B, Moser EI (2007) Pattern separation in the dentate gyrus and CA3 of the hippocampus. Science 315:961-966.

Leutgeb S, Leutgeb JK, Treves A, Moser M-B, Moser EI (2004) Distinct ensemble codes in hippocampal areas CA3 and CA1. Science 305:1295-1298.

Leutgeb S, Leutgeb JK, Barnes CA, Moser EI, McNaughton BL, Moser M-B (2005) Independent codes for spatial and episodic memory in hippocampal neuronal ensembles. Science 309:619-623.

Lindecke A, Korte M, Zagrebelsky M, Horejschi V, Elvers M, Widera D, Prullage M, Pfeiffer J, Kaltschmidt B, Kaltschmidt C (2006) Long-term depression activates transcription of immediate early transcription factor genes: involvement of serum response factor/Elk-1. Eur J Neurosci 24:555-563.

Liu P, Bilkey DK (2002) The effects of NMDA lesions centered on the postrhinal cortex on spatial memory tasks in the rat. Behav Neurosci 116:860-873

Lörincz A, Buzsáki G (2000) Two-phase computational model training long-term memories in the entorhinal-hippocampal region. Ann NY Acad Sci 911:83-111.

Maguire EA, Mummery CJ, Buchel C (2000) Patterns of hippocampalcortical interaction dissociate temporal lobe memory subsystems. Hippocampus 10:475-482.

Maviel T, Durkin TP, Menzaghi F, Bontempi B (2004) Sites of neocortical reorganization critical for remote spatial memory. Science 305:96-99.

McClelland JL, Goddard NH (1996) Considerations arising from a complementary learning systems perspective on hippocampus and neocortex. Hippocampus 6:654-665.

McIntosh AR (1999) Mapping cognition to the brain through neural interactions. Memory 7:523-548.

McIntosh AR, Gonzalez-Lima F (1991) Structural modeling of functional neural pathways mapped with 2-deoxyglucose: effects of acoustic startle habituation on the auditory system. Brain Res 547:295-302.

McNaughton BL, Morris RGM (1987) Hippocampal synaptic enhancement and information storage within a distributed memory system. Trends Neurosci 10:408-415.

Naber PA, Witter MP, Lopes Da Silva FH (2000) Networks of the hippocampal memory system of the rat: the pivotal role of the subiculum. Ann NY Acad Sci 911:392-403.

Nitz D, McNaughton B (2004) Differential modulation of CA1 and dentate gyrus interneurons during exploration of novel environments. J Neurophysiol 91:863-872.

O’Reilly RC, McClelland JL (1994) Hippocampal conjunctive encoding, storage, and recall: avoiding a trade-off. Hippocampus 4:661-682.

Olton DS, Papas BC (1979) Spatial memory and hippocampal function. Neuropsychologia 17:669-682.

Olton DS, Samuelson RJ (1976) Remembrance of places passed: spatial memory in rats. J Exp Psychol Anim Behav Process 2:92-116.

Paxinos G, Watson C (1997) The rat brain in stereotaxic coordinates. San Diego: Academic.

Pothuizen HHJ, Zhang W-N, Jongen-Relo AL, Feldon J, Yee BK (2004) Dissociation of function between the dorsal and the ventral hippocampus in spatial learning abilities of the rat: a within-subject, within-task comparison of reference and working spatial memory. Eur J Neurosci 19:705-712.

Protzner AB, McIntosh AR (2006) Testing effective connectivity changes with structural equation modeling: what does a bad model tell us? Hum Brain Mapp 27:935-947.

Redish AD, Touretzky DS (1997) Cognitive maps beyond the hippocampus. Hippocampus 7:15-35.

Rekart JL, Sandoval CJ, Bermudez-Rattoni F, Routtenberg A (2007) Remodeling of hippocampal mossy fibers is selectively induced seven days after the acquisition of a spatial but not a cued reference memory task. Learn Mem 14:416-421.

Remondes M, Schuman EM (2004) Role for a cortical input to hippocampal area CA1 in the consolidation of a long-term memory. Nature 431:699-703.

Renaudineau S, Poucet B, Save E (2007) Hippocampal place cells firing in zif268 KO mice. In: 39th Annual European Brain and Behaviour Society Abstracts. Neural Plast 2007:23250.

Richardson CL, Tate WP, Mason SE, Lawlor PA, Dragunow M, Abraham WC (1992) Correlation between the induction of an immediate early gene, zif/268, and long-term potentiation in the dentate gyrus. Brain Res 580:147-154.

Richter-Levin G, Thomas KL, Hunt SP, Bliss TVP (1998) Dissociation between genes activated in long-term potentiation and in spatial learning in the rat. Neurosci Lett 251:41-44.

Rolls ET (1996) A theory of hippocampal function in memory. Hippocampus 6:601-620.

Rolls ET, Kesner RP (2006) A computational theory of hippocampal function, and empirical tests of the theory. Prog Neurobiol 79:1-48.

Ros J, Pellerin L, Magara F, Dauguet J, Schenk F, Magistretti PJ (2006) Metabolic activation pattern of distinct hippocampal subregions during spatial learning and memory retrieval. J Cereb Blood Flow Metab 26:468-477.

Steward O, Scoville SA (1976) Cells of origin of entorhinal cortical afferents to the hippocampus and fascia dentata of the rat. J Comp Neurol 169:347-370.

Swanson LW, Sawchenko PE, Cowan WM (1981) Evidence for collateral projections by neurons in Ammon's horn, the dentate gyrus, and the subiculum: a multiple retrograde labeling study in the rat. J Neurosci 1:548-559.

Tabachnik BG, Fidell LS (1996) Using multivariate statistics, Ed 3. New York: HarperCollins.

Tischmeyer W, Grimm R (1999) Activation of immediate early genes and memory formation. Cell Mol Life Sci 55:564-574.

Treves A, Rolls ET (1992) Computational constraints suggest the need for two distinct input systems to the hippocampal CA3 network. Hippocampus 2:189-199.

Vago DR, Bevan A, Kesner RP (2007) The role of the direct perforant path input to the CA1 subregion of the dorsal hippocampus in memory retention and retrieval. Hippocampus 17:977-987.

van Groen T, Wyss JM (1990) Extrinsic projections from area CA1 of the rat hippocampus: olfactory, cortical, subcortical, and bilateral hippocampal formation projections. J Comp Neurol 302:515-528.

van Groen T, Wyss JM (2003) Connections of the retrosplenial granular b cortex in the rat. J Comp Neurol 463:249-263.

Vann SD, Brown MW, Erichsen JT, Aggleton JP (2000) Fos imaging reveals differential patterns of hippocampal and parahippocampal subfield activation in rats in response to different spatial memory tests. J Neurosci 20:2711-2718.

Wilson MA, McNaughton BL (1993) Dynamics of the hippocampal ensemble code for space. Science 261:1055-1058.

Wisden W, Errington ML, Williams S, Dunnett SB, Waters C, Hitchcock D, Evan G, Bliss TVP, Hunt SP (1990) Differential expression of immediate early genes in the hippocampus and spinal cord. Neuron 4:603-614.

Witter MP, Wouterlood FG, Naber PA, Van Haeften T (2000) Anatomical organization of the parahippocampal-hippocampal network. Ann NY Acad Sci 911:1-24.

Zangenehpour S, Chaudhuri A (2002) Differential induction and decay curves of c-fos and zif268 revealed through dual activity maps. Mol Brain Res 109:221-225. 